\title{
INSTABILITY WAVE CONTROL IN TURBULENT JET BY ACOUSTICAL AND PLASMA ACTUATORS
}

\author{
V. F. Kopiev ${ }^{1}$, I. V. Belyaev ${ }^{1}$, G. A. Faranosov ${ }^{1}$, \\ V. A. Kopiev ${ }^{1}$, N. N. Ostrikov ${ }^{1}$, M. Yu. Zaytsev ${ }^{1}$, \\ Yu. S. Akishev ${ }^{2}$, M. E. Grushin ${ }^{2}$, N. I. Trushkin ${ }^{2}$, \\ V. A. Bityurin ${ }^{3}$, A. I. Klimov ${ }^{3}$, I. A. Moralev ${ }^{3}$, I. A. Kossyi ${ }^{4}$, \\ N. K. Berezhetskaya ${ }^{4}$, and M. I. Taktakishvili ${ }^{4}$ \\ ${ }^{1}$ Central Aerohydrodynamics Institute (TsAGI) \\ 17 Radio Str., Moscow 105005, Russia \\ ${ }^{2}$ Troitsk Institute for Innovation and Fusion Research (TRINITI) \\ 12 Pushkovykh Str., Troitsk 142190, Moscow Region, Russia \\ ${ }^{3}$ Joint Institute for High Temperatures \\ Russian Academy of Sciences \\ 13-2 Izhorskaya Str., Moscow 125412, Russia \\ ${ }^{4}$ General Physics Institute \\ Russian Academy of Sciences \\ 38 Vavilova Str., Moscow 119991, Russia
}

\begin{abstract}
It was recently demonstrated by direct experiment in subsonic jets that an instability wave in jet shear layer generated by pure-tone acoustic excitation could be suppressed by another acoustic excitation, which generates an instability wave with the same properties. It was suggested that instability waves could be generated by any oscillating field near the nozzle exit. This paper presents the results of experimental investigations of instability wave suppression by other types of periodic excitation. Three types of plasma actuators are considered: $(i)$ high-frequency $(\mathrm{HF})$ dielectric barrier discharge (DBD); (ii) slipping discharge; and (iii) corona discharge. Control authority of the plasma actuators over instability waves is demonstrated. For high-speed hot jets where instability waves are the dominant noise source, instability wave control is equivalent to noise control.
\end{abstract}

\section{INTRODUCTION}

The paper deals with the problem of instability wave control in turbulent jets. Noise of a turbulent jet issuing from a jet engine nozzle still remains an im- 
portant source of community noise due to civil aircraft. A promising way to reduce jet noise consists in development of active noise control systems [1-3]. The main problems with this idea are related to the lack of understanding of jet noise mechanisms, which could be acted upon by the active closed-loop control system. One of the best understood mechanisms from the standpoint of theoretical description and, hence, most convenient for designing active noise control systems is the mechanism of sound radiation by instability waves that is realized in high-speed jets [4-8].

Although instability waves are also present in low-speed jets, they do not radiate sound directly. Nevertheless, instability wave development at the initial part of the shear layer is essentially the same both for low- and high-speed (including supersonic) jets [4,7]. This implies that successful realization of instability wave control in low-speed jets, which are more convenient for experimental investigation, will confirm the possibility of noise control in jets of technological interest.

In $[9,10]$, the possibility of active instability wave control was theoretically studied within the framework of relatively simple model problems. It has been shown that the instability wave excited by time-harmonic action with fre-

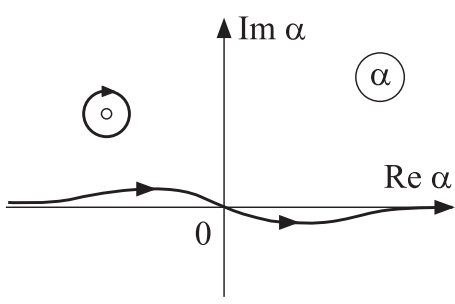

Figure 1 Integration contour on the complex plane $\alpha$ quency $\omega$ can be suppressed by a plane acoustic wave with properly chosen amplitude and phase.

Indeed, from the mathematical point of view, the instability wave excited in a jet by any kind of (small) time harmonic action (e.g., acoustic excitation) corresponds to pole $\alpha_{0}$ of the dispersion relation of the system (mean flow) [11]. For any type of time-harmonic action with frequency $\omega$, the solution for the jet response to the action contains two different parts: the diffraction part related to the integral over real wave-numbers (in terms of inverse Fourier transform) and the hydrodynamic part, i. e., instability wave, associated with the residue at the instability pole $\alpha_{0}$ (Fig. 1); the spatial structure of the hydrodynamic part is thus independent of the way of excitation. Therefore, if an instability wave is excited, it could be suppressed by any other type of excitation, e.g., due to plasma actuators (Fig. 2). Thus, the idea of instability wave control seems quite realizable in theory.

The present paper is devoted to experimental investigation of the feasibility to suppress artificial excited instability wave by means of acoustic as well as plasma actuators. It should be noted that the case in point is the suppression of the hydrodynamic (instability) part of the field, while the remaining acoustic part in general does not vanish and represents a linear combination of the two different diffracted acoustic fields. 


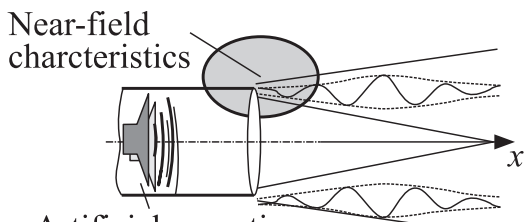

Artificial acoustic excitation

(a)

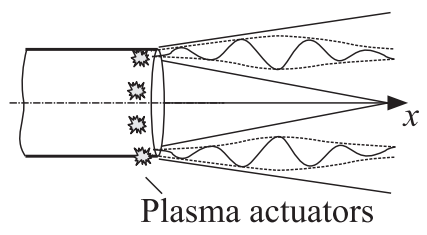

(b)

Figure 2 Artificial instability wave generated by an acoustic sound driver located in the stilling chamber $(a)$ and plasma actuators generating the instability wave with the opposite phase $(b)$

\section{EXPERIMENTAL SETUP}

The experiments were carried out in anechoic chamber AC-2 of TsAGI. The ambient air is compressed and stored in 22 cylindrical tanks at a pressure of up to $22 \mathrm{MPa}$ with a capacity of $9 \mathrm{~m}^{3}$. Jet velocity reached $300 \mathrm{~m} / \mathrm{s}$ for a nozzle diameter of $5 \mathrm{~cm}$. The experiments have been carried out for two jet velocities: 100 and $200 \mathrm{~m} / \mathrm{s}$ (Reynolds number based on the jet diameter was up to $8.3 \cdot 10^{5}$ ). Turbulent velocity fluctuations in the jet potential core were about $1 \%$.

The major experimental tool for instability wave diagnostics in excited jets was two-dimensional (2D) Time-Resolved Particle Image Velocimeter (TR PIV) with the integrated software-hardware system FlowMaster HS-PIV by LaVision $^{\circledR}$. This measurement system can provide quantitative measurements of the instantaneous flow velocity field of turbulent flows with high spatial and temporal (up to $10 \mathrm{kHz}$ ) resolution. The main components and capabilities of FlowMaster are as follows:

- Nd:YAG double-cavity Laser: wavelength $532 \mathrm{~nm}$, pulse energy $2 \times 22.5 \mathrm{~mJ}$ at $1000 \mathrm{~Hz}$, maximum pulse frequency $2 \times 10 \mathrm{kHz}$;

- Digital camera HighSpeedStar 8 with CMOS (complementary metaloxide-semiconductor) matrix $1024 \times 1024$, sampling frequency $7500 \mathrm{fps}$ at full resolution, dynamic range 12 bit, memory $8 \mathrm{~Gb}$;

- Synchronization module HSC Controller with pulse generator for PIV;

- Light sheet optics with light-guide manipulator Laser Guiding Arm;

- 2D PIV Software package: DaVis 8, 2D PIV High-Speed Data Analysis;

- Large Seeding Device (40 jets, for high mass flow rates). Seeding liquid DEHS (Di-2-Ethylhexyl-Sebacat), particle diameter $1 \mu \mathrm{m}$; and

- GPU (Graphics Processing Unit) for acceleration of PIV postprocessing. 
The plane of measurement and, thus, the laser sheet were fixed along the jet axis. The measurement area had dimensions $300 \times 300 \mathrm{~mm}$. This square area was vertically centered on the jet axis. With this setup, it was intended to measure development of coherent structures up to 6 nozzle diameters downstream. The PIV image processing was performed with a multipass interrogation window algorithm $(\mathrm{DaVis} 8)$ with final interrogation window $16 \times 16$ pixel and 50 percent overlapping. Two adjacent velocity vectors were, thus, separated by approximately $2 \mathrm{~mm}$.

\subsection{Instability Wave Identification for an Acoustic and Plasma Forced Jet with Time-Resolved Particle Image Velocimeter}

For quantitative diagnostics of artificially excited coherent structures/instability waves in jets, phase-locked PIV has been used. The method is similar to stroboscopic PIV utilized by Schram et al. [12] for investigation of sound field generated by vortex pairing in a subsonic jet. The principal idea of phase-locked PIV consists in averaging PIV results over a sequence of images with the same phase shift relative to the excitation signal. This allows to separate a periodic (in time) component of velocity field from uncorrelated pulsations. For the purpose of coherent structure identification, a specific value of this phase shift is of little interest because it is well known from schlieren visualization [13] that stroboscopic visualization with frequency multiple to the excitation signal frequency provides a 'frozen' picture of coherent structures in a jet for any phase shift due to the effect of optical averaging. Therefore, PIV measurements have been performed without prescribing this phase shift and, thus, without using a triggering signal from the acoustic excitation; the only requirement was that the excitation frequency has to be equal to the TR PIV frame rate.

\subsubsection{Acoustic excitation}

The TR PIV technique has been applied to an experimental study of high-speed turbulent jets issuing with a velocity of $300 \mathrm{~m} / \mathrm{s}$ from a conical nozzle with an exit diameter of $50 \mathrm{~mm}$. Artificial instability waves were generated by a loudspeaker operating at a discrete frequency and located at $\sim 1.5 \mathrm{~m}$ upstream the nozzle exit. The stability of excitation frequency has been controlled with $1 / 2$-inch microphone B\&K 4189C in the far-field and the data acquisition and analyzing system B\&K Pulse 3560D with an accuracy of $1 \mathrm{~Hz}$.

Typical scalar fields of $V_{x}$ (velocity component along the jet direction) and $V_{y}$ (velocity component normal to the jet direction) obtained in the longitudinal cross section of the jet by averaging a sequence of 300 instantaneous maps are presented in Fig. 3 (here and below, nozzle exit center is located at point $(0 ; 0)$ ). 

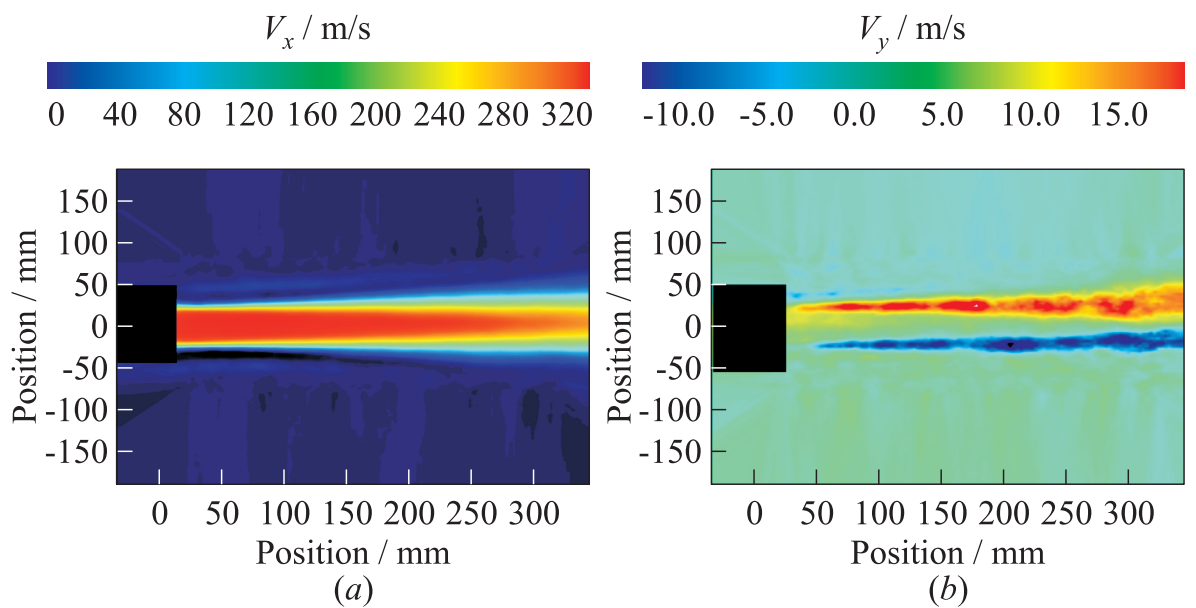

Figure 3 Average $V_{x}(a)$ and $V_{y}(b)$ velocity fields for unforced jet. The black geometrical mask is somewhat larger than the nozzle, which exit is located at $x=0 \mathrm{~mm}$

The results of phase-locked PIV measurements presented in Figs. 4 and 5 demonstrate the appearance of periodic spatial structures (particularly, in Fig. $5 b$ for $V_{y}$ field) which can be associated with the most excited instability wave for this high-speed jet. In the case of mismatch between acoustical excitation frequency (e.g., $3 \mathrm{kHz}$ ) and PIV sampling frequency (e.g., $3.72 \mathrm{kHz}$ ), the orderly structure of velocity field

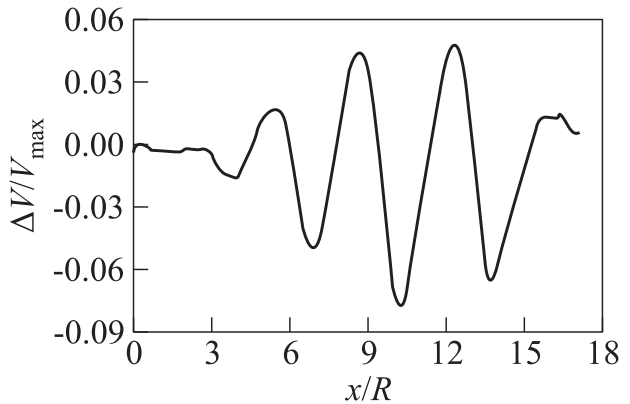

Figure 4 The PIV-measured wavepacket in the jet shear layer were found to disappear, so that the flow picture looks like that for the unforced jet (see Fig. 3). Thus, the experimental method is effective and can be used for identification and analyses of instability waves.

\subsubsection{Plasma actuators for instability waves excitation}

Similar tests have been carried out for the jet excited by electric discharges. For instability wave control in the jet shear layer near to the nozzle exit, several plasma actuators were developed and manufactured. These actuators are 
$V_{x} / \mathrm{m} / \mathrm{s}$

$0 \quad 40 \quad 80120160200240280320$

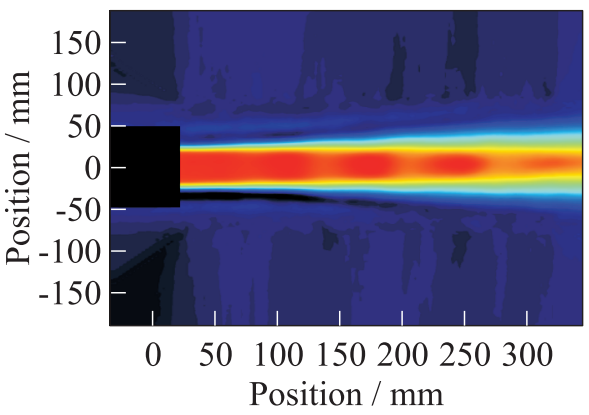

(a)
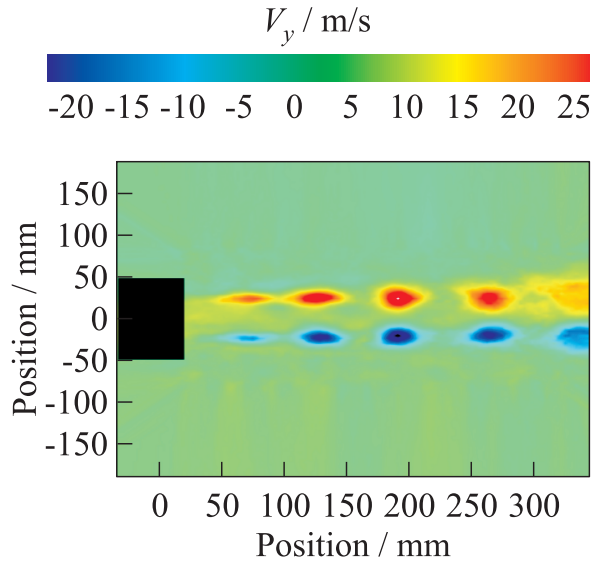

(b)

Figure 5 Velocity fields $V_{x}(a)$ and $V_{y}(b)$ for the jet, acoustically forced at a frequency of $3000 \mathrm{~Hz}$; sampling rate is $3000 \mathrm{fps}$

based on different types of gas discharges (HF DBD, high-current slipping surface discharge, surface barrier corona discharge). For comparability of the received results, identical ceramic nozzles (Fig. 6) with Witoszynski profile (exit diameter $50 \mathrm{~mm}$ ) were used for all actuators.

Below, plasma actuator models for instability wave excitation in turbulent jet shear layer will be presented.

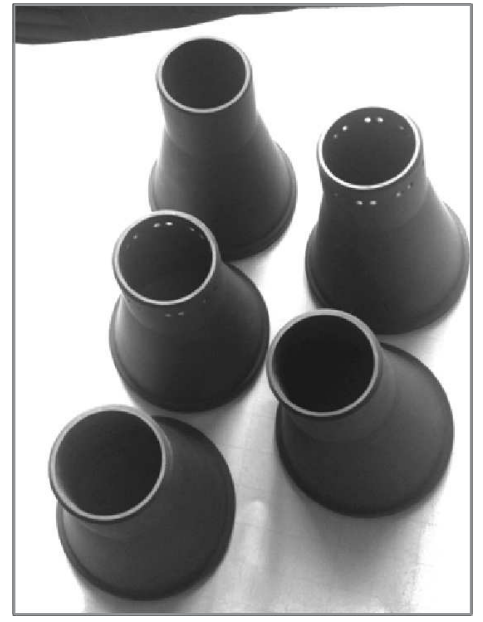

Figure 6 Ceramic nozzles for different types of plasma actuators

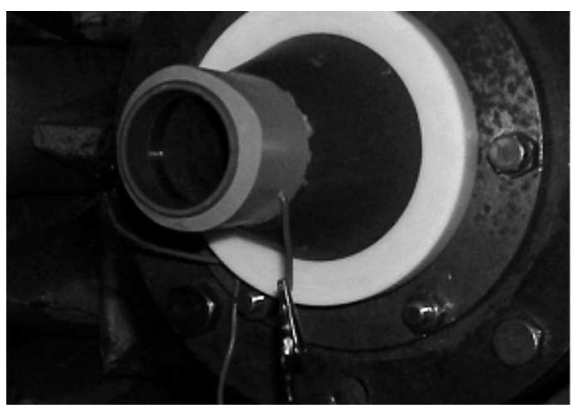

Figure 7 The HF DBD plasma actuator in anechoic chamber AC-2 


\subsection{High-Frequency Dielectric Barrier Discharge Plasma Actuator}

The first actuator uses the classical two-electrode surface DBD scheme [14]. For its operation, a high-voltage, HF power supply was used, which has the frequency range up to $300 \mathrm{kHz}$. Circular electrodes made of aluminum bands were glued on the inner surface of the nozzle (Fig. 7). The external electrode is completely covered with dielectric (silicone compound), which prevented plasma generation on the external nozzle surface while supplying the voltage up to $20 \mathrm{kV}$ to the electrodes. The internal plasma-generating electrode is located at a distance of $5 \mathrm{~cm}$ upstream from the nozzle exit. The discharge develops in the downstream direction.

Electrical parameters of the plasma actuator power supply, designed and built in-house, are as follows:

- high voltage - up to $20 \mathrm{kV}$;

- oscillation frequency — within 100-300 kHz;

- modulation frequency - up to $20 \mathrm{kHz}$;

- modulation depth - 100\%; and

- power - up to $1 \mathrm{~kW}$.

\subsection{Barrier Corona Discharge Plasma Actuator}

The barrier corona discharge plasma actuator allows one to take basic advantages from both corona discharge and DBD [15]. Such discharge combination enables the distance between neighboring needles (pins) to be drastically diminished without giving rise to spark formation. Besides, this design allows unlimited scaling in the transverse direction and enables generation of transverse uniform electric wind.

The sketch of the electrode configuration of a single-disk actuator based on the sectioned DBD surface corona discharge is shown in Fig. 8. External diameter of the disk is equal to $151 \mathrm{~mm}$, internal diameter is $60 \mathrm{~mm}$, and thickness of the disk is $3.5 \mathrm{~mm}$. The disk is made of quartz. There are 36 needle highvoltage (HV) electrodes uniformly distributed over the disk perimeter and connected to

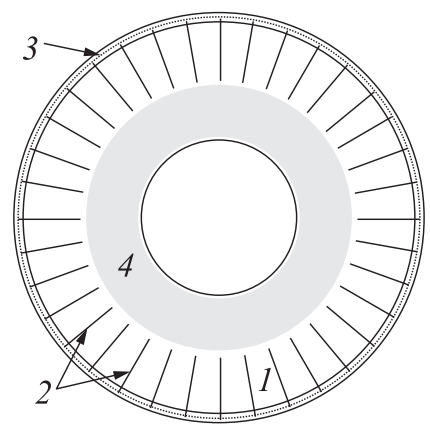

Figure 8 The sketch of the electrode configuration of the singledisk plasma actuator: 1 - quartz disk; 2 - 36 needle electrodes; 3 - HV electrode; and 4 - aluminum foil covering the opposite side of the disk (grounded electrode) a single HV electrode. On the other-side of 


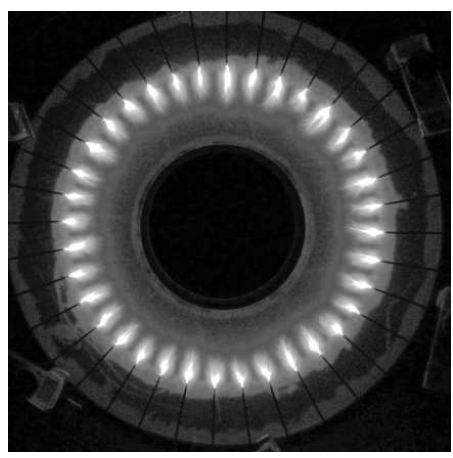

(a)

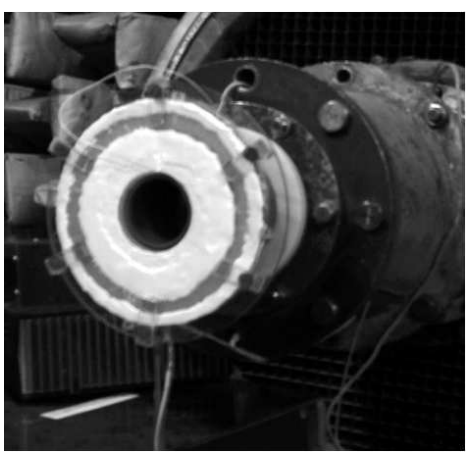

(b)

Figure 9 The images of the disk actuator at operating conditions with switchedon barrier corona discharge: ( $a$ ) the plasma actuator with a single disk; and $(b)$ the dual-disk plasma actuator in anechoic chamber AC-2 of TsAGI

the disk, there is a disk-shaped grounded electrode. The dual-disk plasma actuator consists of two single-disk actuators, which are coaxial and placed faceto-face. The gap between disks in the dual-disk actuator equals to $2.5 \mathrm{~mm}$ (this distance can be varied). The position of plasma actuator at the nozzle edge can be also varied (Fig. 9).

The photos of the single disk plasma actuator in operating conditions and the dual-disk plasma actuator are shown in Figs. $9 a$ and $9 b$, respectively. The actuator is installed on the Witoszynski nozzle in AC-2 anechoic chamber of TsAGI.

This electric discharge was created by the HV generator (designed and built in-house) with the following pulse parameters:

- high voltage - up to $10 \mathrm{kV}$;

- oscillation frequency - $100 \mathrm{kHz}$;

- modulation frequency - up to $1 \mathrm{kHz}$;

- modulation depth - 100\%; and

- power $-<0.6 \mathrm{~kW}$.

\subsection{Slipping Surface Discharge Plasma Actuator}

The slipping surface discharge actuator is shown schematically in Fig. 10.

This discharger consists of a sequence of electrodes and interelectrode gaps located on the outer surface of a flat or cylindrical dielectric base. All electrodes with the exception of two boundary ones (the first and the last) are under the 'floating' potential. 


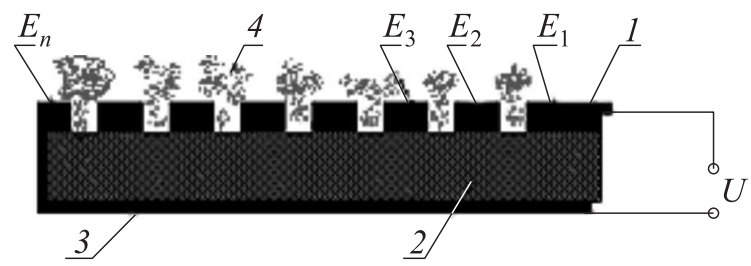

Figure 10 Scheme of the multielectrode discharge actuator: 1 - metallic electrodes $\left(E_{1}, E_{2}, \ldots, E_{n}\right) ; 2$ - dielectric base; 3 - back-current conductor; and 4 - discharge plasma

Dischargers are made in such a way that a high-voltage pulse is initially applied to the first interelectrode gap. After the breakdown in this gap and plasma formation that shorts out the first interelectrode gap, almost entire voltage is applied to the next (second) gap and so on up to the last one. By such means, it is possible to short out with the relatively small voltage an unusually long multielectrode line. To realize this, the configuration of elements and their materials should be chosen in such a way that a relation $C_{1 n} / C_{2 n} \gg 1$ has to be satisfied for the distributed capacities of discharger constructive elements (here, $C_{1 n}$ and $C_{2 n}$ are the distributed capacities between the neighbor electrodes and between electrodes and the back-current conductor, respectively). This discharge was described elsewhere [16].

Slipping surface discharge plasma actuator has eight plasma forming spark gaps on the inner nozzle surface at 1-centimeter distance upstream from the nozzle edge (Fig. 11).

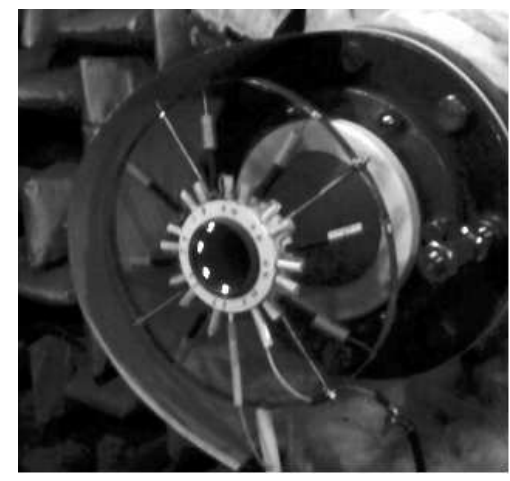

(a)

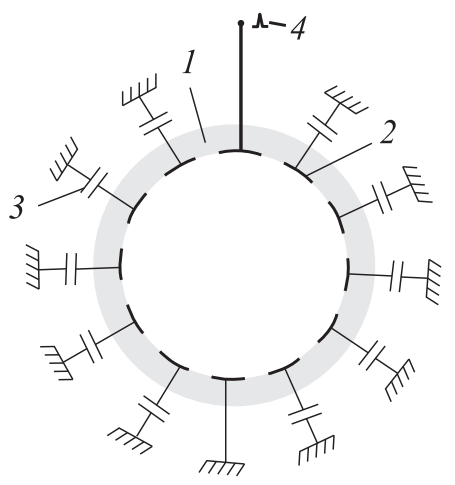

(b)

Figure 11 Photo of the actuator assembled on the ceramic nozzle $(a)$ and its electrical schematic diagram (b): 1 - dielectric nozzle; 2 - electrodes; 3 - capacities $C_{2 n}$; and $4-\mathrm{HV}$ pulse 
This electric discharge was created by the custom-built HV pulse generator with the following pulse parameters:

- mean power $-<0.3 \mathrm{~kW}$;

- voltage - 10-20 kV;

- pulse duration $-\sim 1 \mu \mathrm{s}$;

- pulse current - 100-200 A; and

- pulse repetition rate $-1-1000 \mathrm{~Hz}$.

The energy deposition was about $\sim 0.05 \mathrm{~J}$ in one discharge pulse.

Results of phase-locked PIV measurements demonstrate the capability of these three plasma actuators (based on HF DBD, on barrier corona discharge, and on multispark slipping surface discharge) to create periodic spatial structure, which can be associated with the most excited instability wave for high-speed jets. Plasma-excited instability wave parameters were close to those that were obtained for acoustic excitation. These plasma actuators were used in the experiments on instability wave control.

\section{EXPERIMENTAL VERIFICATION OF THE FEASIBILITY TO CONTROL THE ARTIFICIALLY EXITED INSTABILITY WAVE BY EXTERNAL ACOUSTIC ACTION}

The main objective of the experiment described below was to demonstrate the feasibility to control artificially excited instability waves in turbulent jets. The TR PIV diagnostic technique described in the previous section was used in the experiments. The idea of the experiment is based on the theory of acoustic instability wave control described in $[10,17]$. In these papers, the instability wave is excited by a plane acoustic wave coming from semiinfinite cylindrical nozzle, and then this instability wave is cancelled by an external acoustic wave with properly chosen amplitude and phase according to the analytical solution of the control problem. The main result of the developed theory consists in justification of the possibility of effective control over artificial instability wave by means of an external action with the amplitude of the same order as the internal one. It should be emphasized here that it is fundamentally important that inner and outer excitations of the jet are considered, since otherwise, e.g., in the case of inner-inner excitation, the problem would reduce to the trivial acoustic cancellation inside the duct, whereas in the case of inner and outer excitations, acoustic fields are not canceled, while the Kelvin-Helmholtz instability is canceled.

In order to test experimentally the possibility of shear layer instability control which has been shown to be realizable theoretically, first, axisymmetric external 

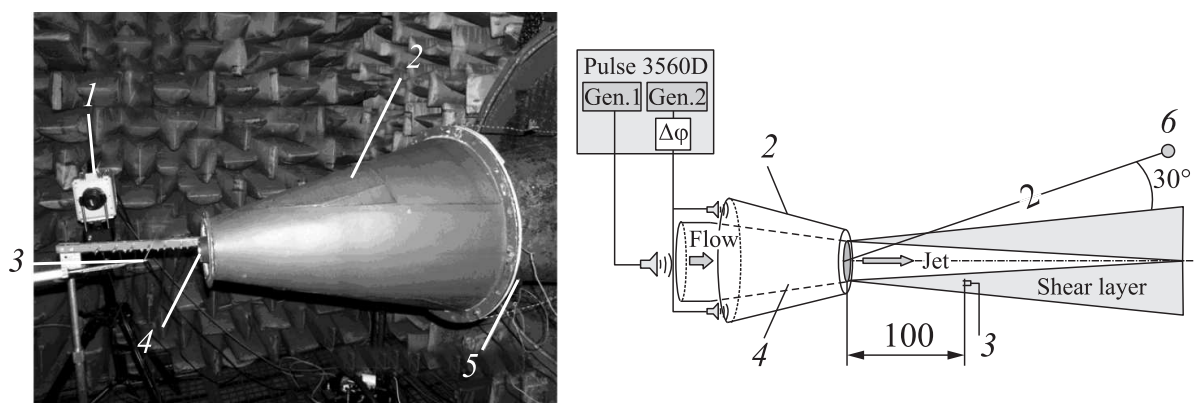

Figure 12 Experimental setup; $(a)$ photo in AC-2; and (b) sketch of the setup: $1-$ PIV camera; 2 - acoustic concentrator; 3 - hot-wire probe; 4 - nozzle; 5 - outer loudspeaker; and 6 - far-field microphone. Dimensions are in millimeters

and internal acoustic excitations of the jet should be provided for generation of a mainly axisymmetric instability (zero mode) that is close to the model problem considered theoretically. Axisymmetric internal excitation was created by a loudspeaker located in the settling chamber at a distance of about $1.5 \mathrm{~m}$ upstream from the nozzle exit, the diameter of the nozzle was $52 \mathrm{~mm}$. Such a configuration was tested to provide azimuthally uniform near- and far-field at the frequency of excitation with an error less than $\pm 0.5 \mathrm{~dB}$. Excitation of an axisymmetric external acoustic wave near the nozzle exit with the amplitude comparable to the internal one turned out to be a more complicated problem. It was resolved by making use of a metal truncated cone aligned with the jet axis and forming a quasi-annular duct around the main nozzle (Fig. 12). The back surface of the cone was closed by a special ring cover with two loudspeakers inserted in it. The exit plane of the cone was coplanar to the main nozzle exit. This system of external excitation provided azimuthal uniformity of the near field with an error of $\pm 0.9 \mathrm{~dB}$. Azimuthal structure of the field was measured by 6 microphones located symmetrically near the front edge of the cone. These microphones were removed during the main measurements performed with $50 \mathrm{~m} / \mathrm{s}$ jet excited at $1 \mathrm{kHz}$ (Strouhal number $\mathrm{St}=1.04$ ).

The measurements were conducted in two stages. During the first stage, a far-field microphone and a hot-wire probe were used (see Fig. 12). The 1/2inch 4189C Bruel\&Kjaer microphone was located in the far field at a distance of $2 \mathrm{~m}$ from the nozzle exit center at an angle of $30^{\circ}$ to the jet axis, and the Dantec 55P01 hot-wire probe was placed approximately in the middle of the shear layer $100 \mathrm{~mm}$ downstream from the nozzle exit plane. During the second stage, the hot-wire probe was removed and PIV measurements of the excited jet were performed.

The microphone and hotwire measurements showed that provided the internal and external excitations are adjusted in the proper way, one can effectively 


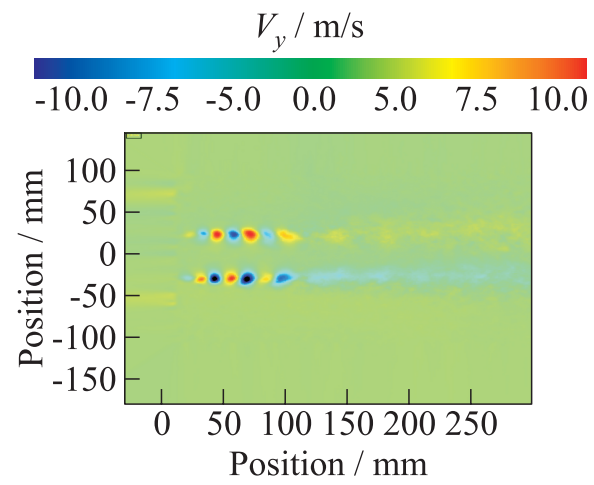

(a)

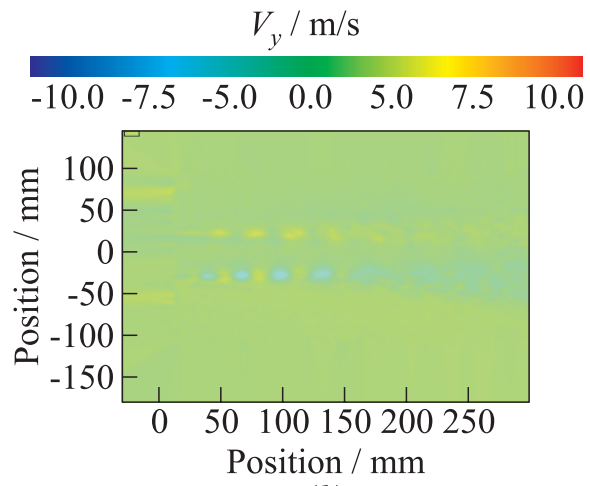

(b)

Figure 13 Radial velocity field $\left(V_{y}\right)$ for the phase shifts corresponding to maximal amplification $(a)$ and maximal attenuation $(b)$ of the instability in the jet shear layer

amplify or attenuate hydrodynamic coherent structures in the jet just by varying the phase shift between the sources of excitation.

However, these measurements pertain to one point where the hot-wire probe is located and, thus, they do not preclude the possibility that change in the measured amplitude is not due to suppression/amplification of instability wave but rather due to shift of the nodes of instability wave. To ensure that the amplitude of instability waves does indeed change in the entire shear layer, phaselock PIV measurements were carried out. These measurements were performed at an excitation frequency of $1 \mathrm{kHz}$, the PIV sampling frequency being the same

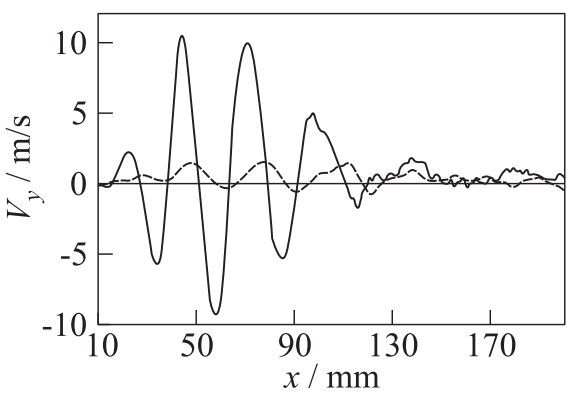

Figure 14 Radial velocity $\left(V_{y}\right)$ profiles along the lip-line for the regimes presented in Fig. 13
$(1 \mathrm{kHz})$ in accordance with the procedure described above. The microphone was removed. The source amplitudes were adjusted by means of hot-wire measurements, then the hotwire probe was also removed from the jet and phase-lock PIV measurements were carried out for different phase shifts between the internal and external sources (with step $30^{\circ}$ ). The evolution of coherent structures observed after PIV measurements postprocessing (its parameters were the a same as in a previous section) is 
presented in Fig. 13, where one can see radial velocity field $\left(V_{y}\right)$ for the phase shifts corresponding to the maximal amplification and maximal attenuation of instability waves. Let notice that these shifts differ by $180^{\circ}$.

In Fig. 14, one can see the profiles of radial velocity $\left(V_{y}\right)$ along the lip-line for the regimes presented in Fig. 13. It is seen from Fig. 14 that in the conducted experiment, a fivefold variation in shear layer instability amplitude takes place. It should be emphasized that it does not seem to matter what particular type of action is used for generation of instability wave. In the described experiment, it was created by an acoustic excitation, but equally well it could be produced by any other actuator.

\section{EXPERIMENTAL VERIFICATION OF THE POSSIBILITY TO CONTROL ARTIFICIAL INSTABILITY WAVE BY PLASMA ACTUATORS}

In the experiments described in this section, plasma actuators have been used instead of external acoustic waves. A sketch of the instability wave control experiments is depicted in Fig. 15.

In this scheme, the 2D TR PIV system described above was also used. Again, the experiments were carried out in anechoic chamber AC-2 of TsAGI. First, the intensities of acoustical and plasma excitation were adjusted so as to give approximately equal levels of instability wave amplitude. For this adjustment, PIV measurements of the amplitude of instability wave generated by the plasma actuator and the loudspeaker have been performed separately. After the acoustical and plasma excitations were adjusted independently to the same level, they were turned on simultaneously with controllable phase shift $\Delta \varphi$ between them.

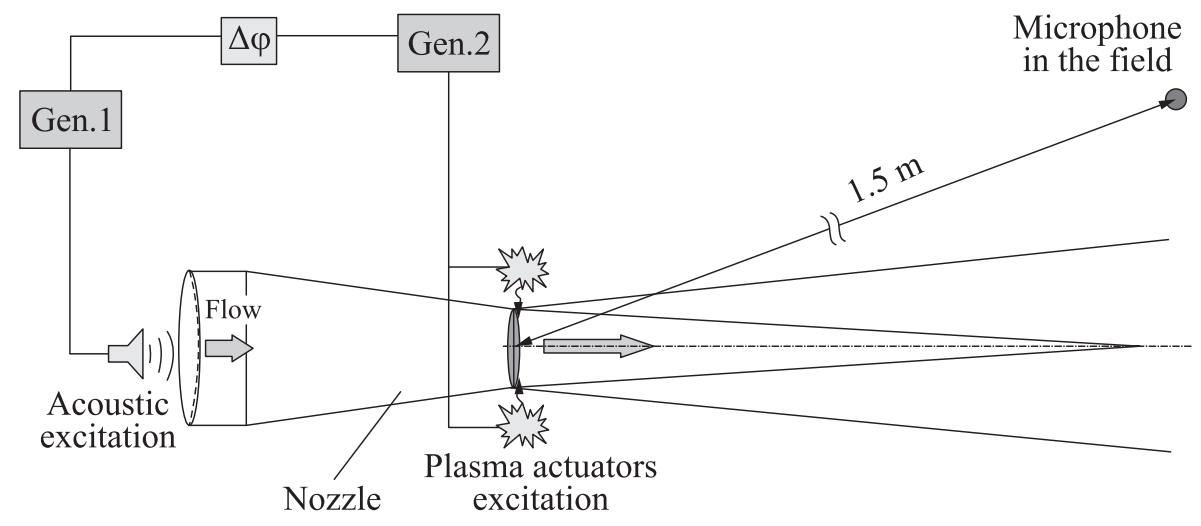

Figure 15 Sketch of instability wave control experiments with plasma actuators 
Variation of the phase shift led to variations of the instability wave amplitude measured by phase-lock TR PIV.

The experiments were performed with three types of plasma actuators described above, the jet with a velocity of $100 \mathrm{~m} / \mathrm{s}$ was excited at a frequency of $1 \mathrm{kHz}$. With the HF DBD actuator, the experiments were also performed for $200 \mathrm{~m} / \mathrm{s}$ jet excited at a frequency of $2 \mathrm{kHz}$. For all these cases, Strouhal number was 0.5 because a particular choice of excitation frequency is of little importance in this study and it was deemed convenient to choose a frequency near the max-

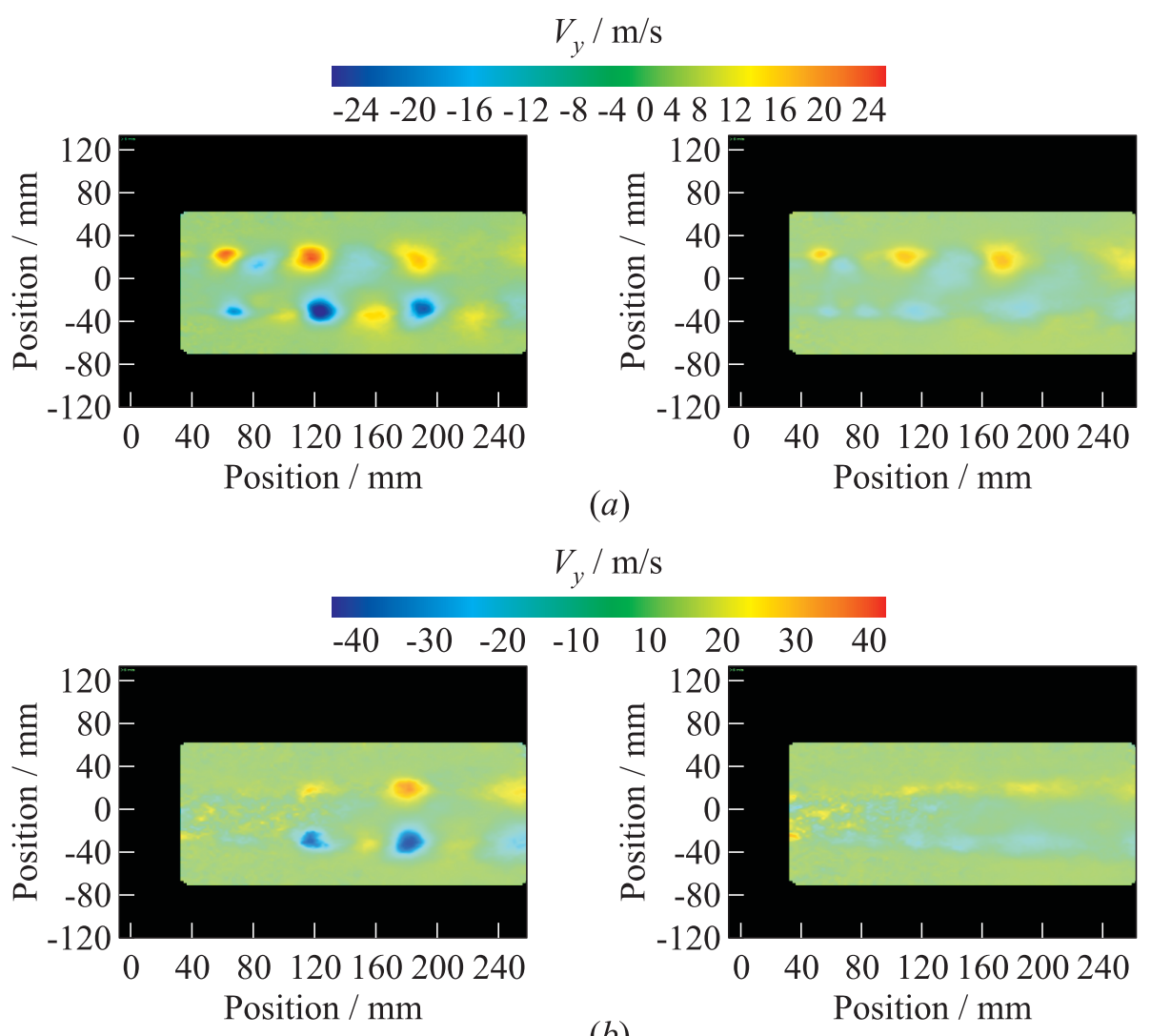

(b)

Figure 16 Combined excitation by the HF DBD plasma actuator and the loudspeaker. The radial velocity field $\left(V_{y}\right)$ for phase shifts corresponding to maximal amplification (left column) and maximal attenuation (right column) of the instability wave in the jet shear layer: $(a)$ jet velocity $100 \mathrm{~m} / \mathrm{s}$, excitation frequency $1 \mathrm{kHz}$; and $(b)$ jet velocity $200 \mathrm{~m} / \mathrm{s}$, excitation frequency $2 \mathrm{kHz}$ 
imum of jet noise spectrum. The same refers to the choice of azimuthal mode $m=0$. Nevertheless, it is worth noting that a study of higher azimuthal modes is of undoubted interest and is going to be carried out in future after appropriate upgrade of the plasma actuators power supply.

Evolution of coherent structures measured by PIV is shown in Figs. 16 and 17, which depict $V_{y}$ velocity fields under the combined action of the loudspeaker and different plasma actuators for two phase shifts between them corresponding to
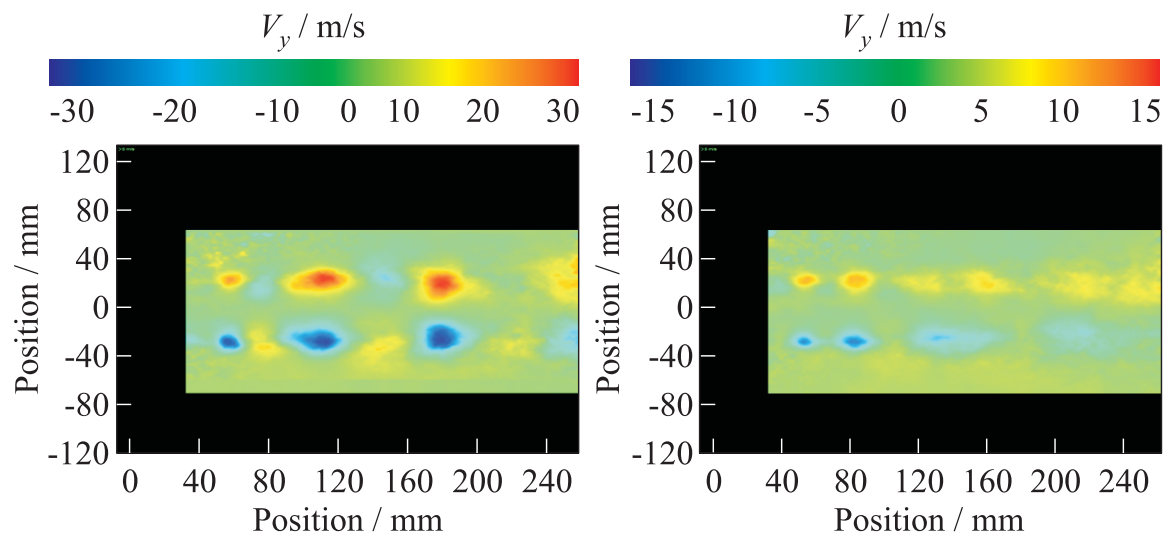

(a)

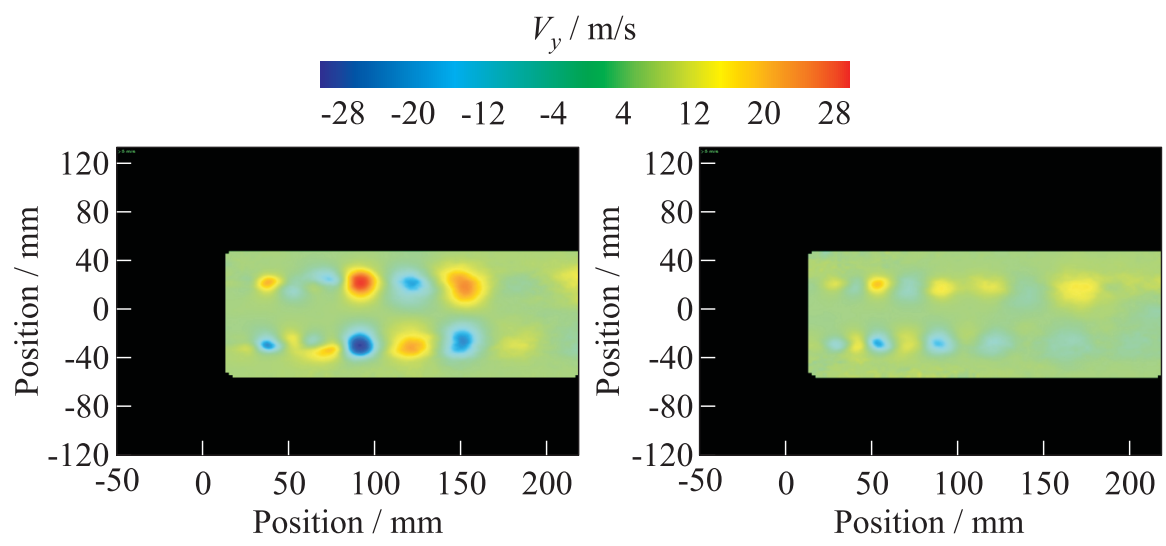

(b)

Figure 17 Combined excitation by the barrier corona discharge plasma actuator and the loudspeaker $(a)$ and by the slipping surface discharge plasma actuator and the loadspeaker $(b)$. The radial velocity field $\left(V_{y}\right)$ for phase shifts corresponding to maximal amplification (left column) and maximal attenuation (right column) of the instability wave in the jet shear layer. Jet velocity $100 \mathrm{~m} / \mathrm{s}$, excitation frequency $1 \mathrm{kHz}$ 


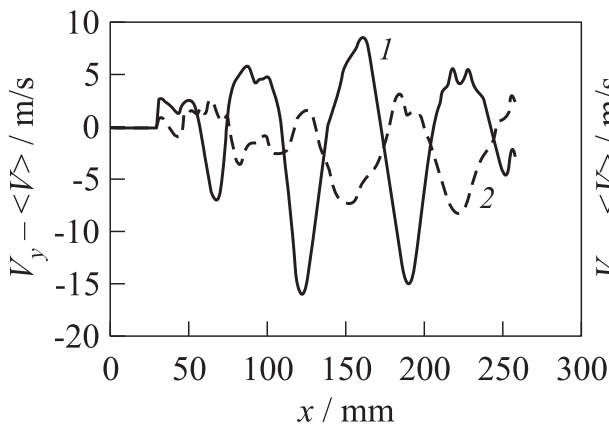

(a)

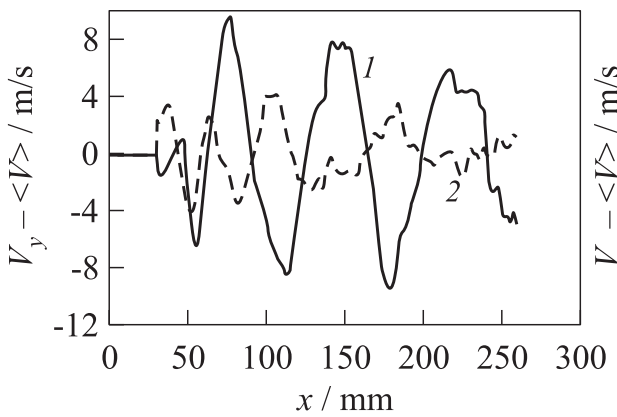

(c)

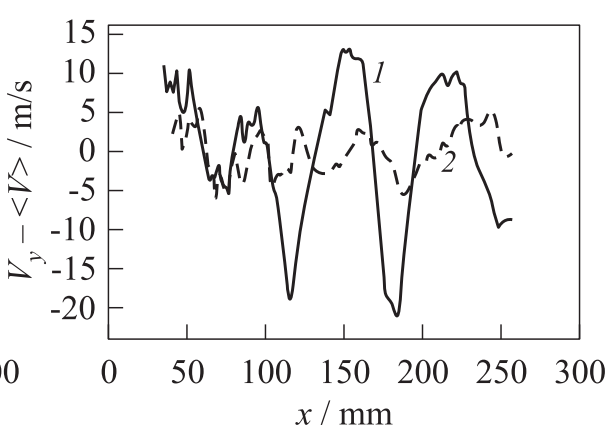

(b)

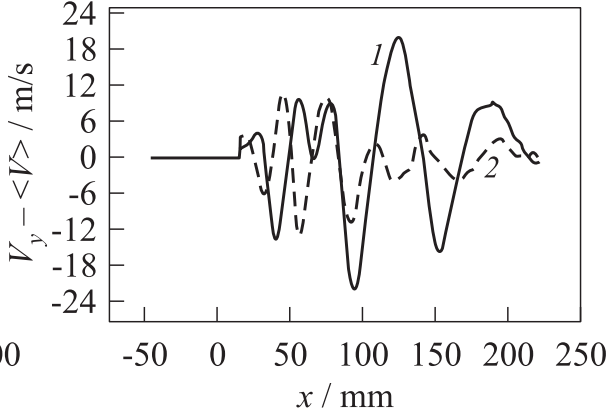

(d)

Figure 18 Radial velocity $\left(V_{y}\right)$ profiles along the lip-line for the regimes shown in Figs. 16 and 17, amplification (1) and attenuation (2): (a) HF DBD plasma actuator for $100 \mathrm{~m} / \mathrm{s}$ jet; (b) HF DBD plasma actuator for $200 \mathrm{~m} / \mathrm{s}$ jet; $(c)$ barrier corona discharge plasma actuator; and $(d)$ slipping surface discharge plasma actuator

maximal amplification (left columns) and maximal attenuation (right columns) of the instability wave amplitude.

From Figs. 16 and 17, one can conclude that phase shift adjustment lead to instability wave mitigation. In Fig. 18, one can see the profiles of radial velocity $\left(V_{y}\right)$ along the lip-line for two phase shifts, which correspond to maximal amplification (1) and maximal attenuation (2).

It is seen from Fig. 18 that the actuators have control authority over instability wave amplitude in the shear layer. The highest level of variation was obtained for the HF DBD plasma actuator and jet velocity $200 \mathrm{~m} / \mathrm{s}$. In this case, the artificial instability wave was suppressed up to the value of radial velocity $\left(V_{y}\right)$ in the unforced jet (compare with Fig. $3 b$ ). Although variation for the plasma actuators (the amplitudes for the most amplified and most attenuated waves differ by a multiple of 4 ) is somewhat smaller than for the acoustic actuator (they differ by a multiple of 5), this should not be regarded as an indication that 
plasma actuators are less effective. The variation in instability wave amplitudes between the most attenuated and most amplified cases is ultimately dependent upon the adjustment of the amplitudes of the inner and external sources, and the adjustment is easier to perform for the acoustic actuator and, thus, can be performed with higher accuracy.

\section{CONCLUDING REMARKS}

In this work, control over artificial instability wave excited by preset acoustical source is realized, which experimentally confirms the theoretical conclusion [9] that instability wave can be cancelled by means of an another external action under condition of a correct choice of its amplitude and phase. The PIV measurements have shown that for the jet considered, the proper choice of the amplitude and phase of control action made on the basis of hot-wire measurements leads to a considerable (fivefold) decrease in instability wave amplitude in the entire shear layer.

The response of subsonic turbulent jet to plasma actuator excitation has also been investigated. Phase-locked PIV measurements have shown that plasma actuator excitation leads to instability waves/vortex rings formation in the jet shear layer for three elaborated types of plasma actuators. The variation of the phase shift between the adjusted acoustical and plasma excited sources gives rise to an approximately sinusoidal variation of instability wave amplitude, so that there exist phase shifts corresponding to maximal amplification and maximal attenuation (shifted by $180^{\circ}$ ) of the coherent structures, and these minimum and maximum amplitudes differ by a multiple of up to 4 depending on the actuator type. The proposed systems of actuators allow effective controlling of artificially excited instability waves in the entire shear layer of turbulent jet. Realization of this effect in high-speed jet where instability waves can directly radiate sound may allow development of active close-loop systems for jet noise mitigation.

\section{ACKNOWLEDGMENTS}

This work was carried out under Coordinated EU-RF FP7 Project No. ACP9GA-2010-266103 ORINOCO and the Ministry of Industry and Trade of Russian Federation (project ORINOCO).

\section{REFERENCES}

1. Maury, R., M. Koenig, L. Cattafesta, P. Jordan, J. Delville, J.-P. Bonnet, and Y. Gervais. 2009. Extremum-seeking optimisation of fluidic jet-noise control. AIAA Paper No. 2009-3132. 
2. Kopiev, V.F., I. V. Belyaev, V.A. Kopiev, N. N. Ostrikov, and G. A. Faranosov. 2011. Instability wave control by plasma actuators: Problems and prospects. AIAA Paper No. 2011-973.

3. Kearney-Fischer, M., J.-H. Kim, and M. Samimy. 2011. Noise control of a high Reynolds number high speed heated jet using plasma actuators. Int. J. Aeroacoustics 10(5-6):491-509.

4. Tam, C.K.W., and D.E. Burton. 1984. Sound generated by instability waves of supersonic flows. J. Fluid Mech. 138:249-295.

5. Suzuki, T., and T. Colonius. 2006. Instability waves in a subsonic round jet detected using a near-field phased microphone array. J. Fluid Mech. 565:197-226.

6. Ryu, J., S. K. Lele, and K. Viswanathan. 2007. Identification of instability waves in high-speed turbulent jets. AIAA Paper No. 2007-3624.

7. Zaitsev, M. Yu., V.F. Kopiev, and S. A. Chernyshev. 2009. Experimental investigation of the role of instability waves in noise radiation by supersonic jets. Fluid Dyn. 44(4):587-595.

8. Morris, P. J. 2010. The instability of high speed jets. Int. J. Aeroacoustics 9(1-2):150.

9. Kopiev, V. F., and G. A. Faranosov. 2008. Control over the instability wave in terms of the two-dimensional model of a nozzle edge. Acoust. Phys. 54(3):319-326.

10. Faranosov, G. A. 2008. Instability wave control in a subsonic round jet. Acoustics'08 Paris Conference Proceedings. 1839-1844.

11. Munt, R. M. 1977. The interaction of sound with a subsonic jet issuing from a semiinfinite cylindrical pipe. J. Fluid Mech. 83(4):609-640.

12. Schram, C., S. Taubitz, J. Anthoine, and A. Hirschberg. 2005. Theoreti$\mathrm{cal} / \mathrm{empirical}$ prediction and measurement of the sound produced by vortex pairing in a low Mach number jet. J. Sound Vib. 281:171-187.

13. Kopiev, V. F., M. Yu. Zaitsev, S. I. Inshakov, and L. P. Guriashkin. 2003. Visualization of the large-scale vortex structures in excited turbulent jets. J. Visual. Japan 6(3):303-311.

14. Kopiev, V.F., V.A. Bityurin, I. V. Belyaev, S. M. Godin, M.Y. Zaitsev, A. I. Klimov, V.A. Kopiev, I. A. Moralev, and N. N. Ostrikov. 2012. Jet noise control using the dielectric barrier discharge plasma actuators. Acoust. Phys. 59(4):473-482.

15. Akishev, Yu. S., G. I. Aponin, V.B. Karal'nik, A. E. Monich, and N.I. Trushkin. 2004. Structure of the surface streamers of an ac barrier corona in argon. Plasma Phys. Rep. 30(12):1012-1026.

16. Anpilov, A. M., E. M. Barkhudarov, N.K. Berezhetskaya, V.A. Kopiev, and I. A. Kossyi. 1998. Source of a dense metal plasma. Plasma Sources Sci. Technol. $7(2): 141-148$.

17. Kopiev, V.F., I. V. Belyaev, M. Yu. Zaytsev, V. A. Kopiev, and G. A. Faranosov. 2013. Acoustical control of instability waves in turbulent jet. Acoust. Phys. 59(1):434-441. 